\title{
A new method to measure necrotic core and calcium content in coronary plaques using intravascular ultrasound radiofrequency-based analysis
}

\author{
Eun-Seok Shin • Hector M. Garcia-Garcia • \\ Patrick W. Serruys
}

Received: 5 October 2009/Accepted: 17 December 2009/Published online: 9 January 2010

(C) The Author(s) 2010. This article is published with open access at Springerlink.com

\begin{abstract}
Although previous intravascular ultrasound (IVUS) radiofrequency-based analysis data showed acceptable reproducibility for plaque composition, measurements are not easily obtained, particularly that of lumen contour, because of the limited IVUS resolution. The purpose of this study was to compare a new measurement method (Shin's method) and the conventional measurement method for necrotic core and calcium content in atherosclerotic lesions using Virtual Histology-intravascular ultrasound (VH-IVUS). Fifty-seven patients with unstable angina who underwent elective percutaneous coronary intervention were included. Shin's method focuses on catheter contour, instead of lumen contour, and vessel contour. Patients ages ranged from 46 to 88 years, and 34 were men. A total of 1,401 frames from 59 culprit lesions were assessed. There were no significant differences in the mean area and volume of necrotic core and dense calcium between the two methods. Correlation coefficients $(R)$ were $\geq 0.99$ for all above mentioned parameters $(P<0.001)$. Between methods, the absolute differences in mean area and volume of necrotic core were
\end{abstract}

E.-S. Shin $(\square)$

Ulsan University Hospital, Thoraxcenter, Erasmus

Medical Center, Ulsan, South Korea

e-mail: sesim98@yahoo.co.kr

H. M. Garcia-Garcia · P. W. Serruys

Thoraxcenter, Erasmus Medical Center, Rotterdam,

The Netherlands
$0.02 \pm 0.02 \mathrm{~mm}^{2}$ and $0.34 \pm 0.29 \mathrm{~mm}^{3}$, respectively, while for mean area and volume of dense calcium, the absolute differences were $0.04 \pm 0.07$ $\mathrm{mm}^{2}$ and $0.36 \pm 0.52 \mathrm{~mm}^{3}$, respectively. The reproducibility of Shin's method was excellent. For area of the necrotic core and dense calcium, the means of the differences between the two measurements were nearly zero, and the reproducibility coefficients were within $1 \%$ of the means of the two measurements. Mean analysis time for both measurements was $26.8 \pm 6.7 \mathrm{~min} / \mathrm{segment}$ in the conventional method and $3.3 \pm 0.6 \mathrm{~min} / \mathrm{segment}$ in Shin's method. Shin's method for measurement of necrotic core and dense calcium using VH-IVUS demonstrated a good correlation with the conventional method and excellent reproducibility. Also, Shin's method required a significantly shorter analysis time than the conventional method. Therefore, Shin's method could replace the conventional method for necrotic core and calcium measurement in atherosclerotic lesions, and it might be useful in the catheterization laboratory for online clinical decision.

Keywords Intravascular ultrasound ·

Necrotic core - Dense calcium - Catheter contour

\section{Introduction}

While there is a well established relationship between degree of stenosis and plaque burden and ischemic 
symptoms in patients with stable angina, plaque composition, especially necrotic core, is more relevant in terms of occurrence of acute coronary syndromes [1-4]. Virtual Histology-intravascular ultrasound (VH-IVUS), which permits the analysis of coronary plaque composition in vivo, is increasingly used in clinical research [5-12]. Despite its demonstrated utility in quantifying coronary plaque components with high predictive accuracy both in vitro and in vivo $[13,14]$, VH-IVUS is infrequently used routinely in the catheterization laboratory for clinical decision making partly because it is not easy to immediately draw the contours. In particular, lumen contour detection takes a long time and it is especially challenging in patients with high risk lesions (i.e., acute coronary syndrome) because there is no clear separation between luminal thrombi and intima. The aim of this study is to assess a new measurement method (Shin's method) that readily provides on-line (i.e., immediately after acquisition) necrotic core and calcium measurements to help interventionalists to better assess coronary lesions irrespective of clinical presentation. Therefore, in the present study we compared the new measurement method (Shin's method) and the conventional measurement method in quantifying necrotic core and calcium in atherosclerotic lesions using VH-IVUS.

\section{Methods}

Study patients

In this study, 1,401 frames and 59 segments of culprit lesions were imaged in a total of 57 consecutive patients with unstable angina who underwent coronary artery stenting. Specifically, measurements of necrotic core and dense calcium (i.e., area and volume) by VH-IVUS were considered. Unstable angina was defined according to the Braunwald classification [15], and patients with class IIB and IIIB were included in the study. Patients were excluded if they had an IVUS-detected thrombotic lesion, left main disease and chronic total occlusion. Culprit lesion is defined as the lesion related to the patient's symptom that was treated with stenting. We determined the analysis times needed for both approaches and report the mean times of analysis. This study was performed with the patient's written informed consent and approval of the institutional review board.

\section{VH-IVUS}

Details of the radiofrequency (RF) IVUS technique have been extensively validated [9, 10, 13, 14]. In brief, spectral analysis of VH-IVUS data is used to create tissue maps to classify atherosclerotic plaques into four major components (fibrous: green; fibrofatty: light-green; dense calcium: white; and necrotic core: red). In this study, only dense calcium and necrotic core were considered because they are commonly found in high-risk plaques; both were assessed using the conventional method and Shin's method.

\section{IVUS data acquisition}

IVUS data were acquired with commercially available phased-array IVUS catheters (Eagle Eye Gold 2.9-F $20 \mathrm{MHz}$, Volcano Corporation, Rancho Cordova, USA) by a dedicated VH-IVUS console (Volcano Corporation, Rancho Cordova, USA). Image acquisition was ECG-gated. After intracoronary injection of $200 \mu \mathrm{g}$ nitroglycerine, continuous pullback of the IVUS catheter was performed using a motorized pullback device at $0.5 \mathrm{~mm} / \mathrm{s}$. Data of both pullbacks were stored on hard disk for off-line analysis.

\section{VH-IVUS data analysis}

RF-IVUS analysis was performed off-line by an independent core laboratory (Cardialysis BV, Rotterdam, The Netherlands). The region of interest comprises the most diseased $10-\mathrm{mm}$ segment within the culprit lesion that was contained the largest plaque burden. This approach mimics a real clinical situation in which the interventionalists must make a clinical decision on the most stenotic segment using not only geometrical but also compositional analysis.

\section{Conventional method of analysis}

The contours were traced using a semiautomatic contour detection program (PcVH 2.2, Volcano Corporation, Rancho Cordova, USA). The contours of each cross sectional image per frame were manually corrected if required to meet a high standard of accuracy. The lumen contour was detected by tracing the leading edge of the intima, and the vessel contour by tracing the leading edge of 

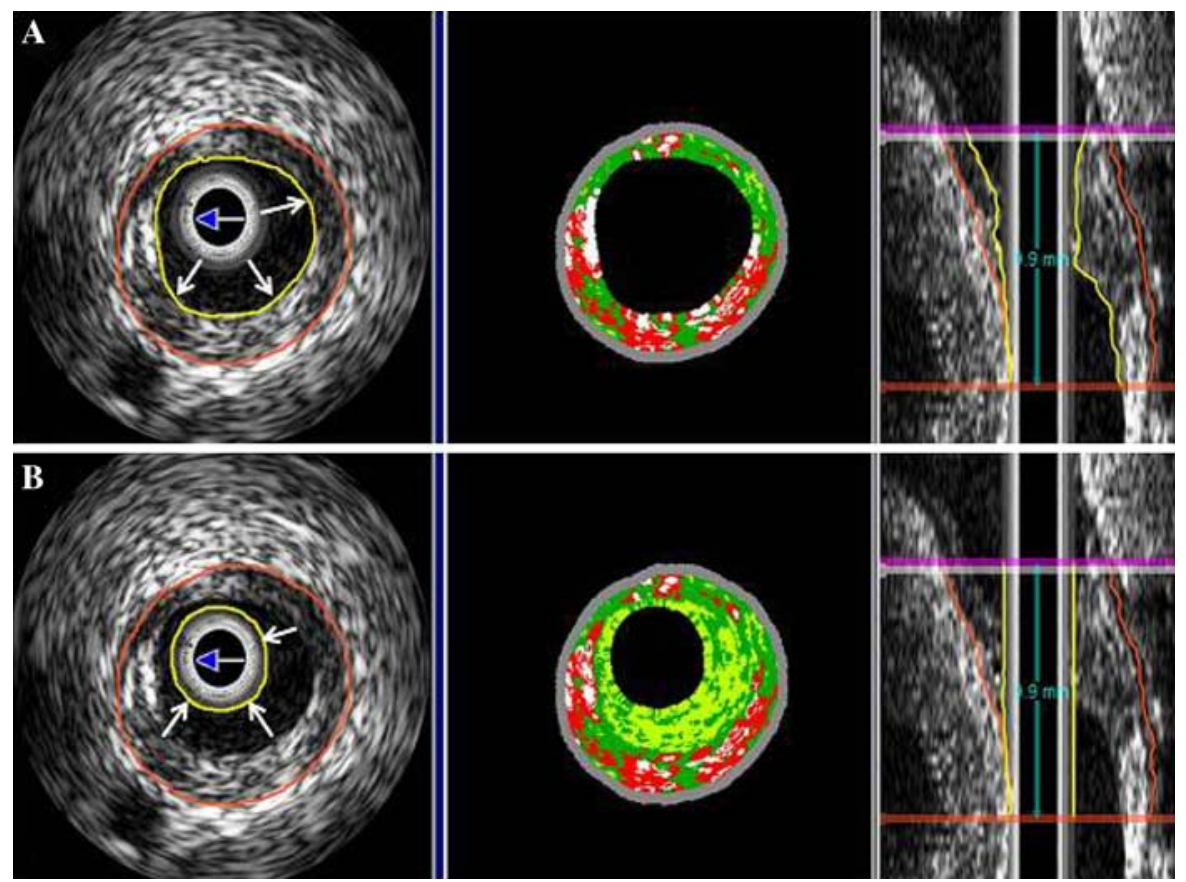

Fig. 1 A The conventional method entails drawing the lumen contour (white arrows). B Instead of measuring the lumen, Shin's method entails drawing behind the catheter. While there is a "flare" (ring-down artifact) around the catheter, the artificial contour must be drawn away from this flair (white arrows)
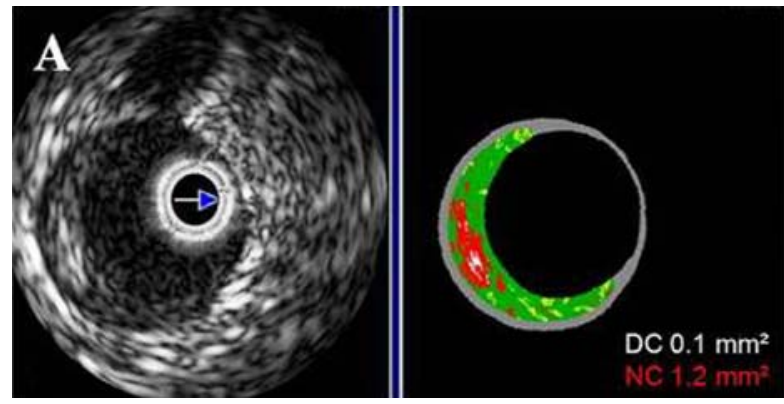

Fig. 2 A and B Corresponding IVUS frames. Shin's method (B) shows similar necrotic core and dense calcium area compared with the conventional method (A). To compare only the influence in terms of dense calcium and necrotic core of the

the adventitia. For each frame geometrical and compositional analyses were performed; the mean area of the vessel and lumen were obtained and for each segment, the vessel and lumen volumes were generated (Figs. 1, 2).

Shin's method of analysis

Shin's method also used the off-line software PcVH 2.2. Instead of measuring the lumen as described
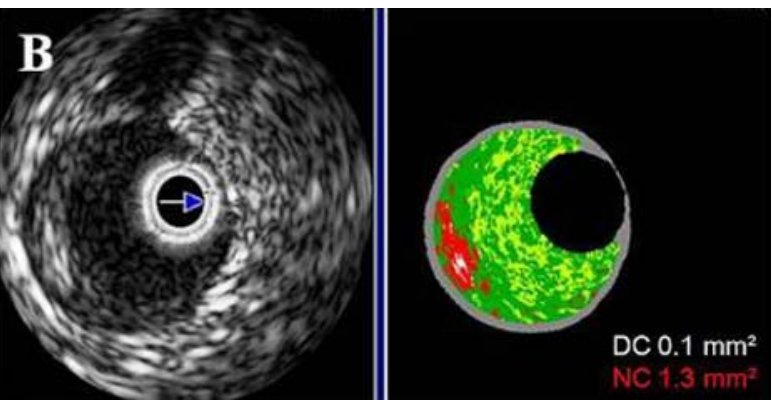

two different approaches the same contour of the vessel was used ( $F I$ fibrous, $F F$ fibrofatty, $D C$ dense calcium, $N C$ necrotic core)

above for the conventional method, in Shin's method the lumen contour is drawn around the IVUS catheter (hereafter termed catheter contour), that is, without following the leading edge of the interface lumen intima. When there is a "flare" (ring-down artifact) around the IVUS catheter, this catheter contour must be drawn away from this flare (Fig. 1). The catheter contour was manually detected. To compare only the influence of the two different approaches (Conventional vs. Shin's), in terms of dense calcium and 
necrotic core, the same contour of the vessel was used

(Figs. 1, 2).

Using Shin's method the following information can be obtained:

1. Necrotic core and dense calcium areas and volumes.

2. Vessel area and volume.

However, using Shin's method the following information can NOT be obtained:

1. Fibrous and fibrofatty areas and volumes.

2. Lumen and plaque areas and volumes.

Data analysis

For all comparisons, two levels were considered, the frame level and the segment level.

First, we compared the two methods (necrotic core and calcium) by one expert analyst. To assess the reproducibility of Shin's method, eight segments (189 frames) were randomly selected and the same segments were analyzed twice by the same analyst with a 3-week time difference. To this end, the vessel contour was kept from the conventional method, so that only the catheter contour was drawn twice.

\section{Statistical analysis}

Analyses were performed with SPSS 16.0 (SPSS Inc., Chicago, IL). Dichotomous data are presented as frequencies. Quantitative data are presented as mean $\pm \mathrm{SD}$ and compared using Student's $t$-test, and linear regression analysis for repeated measures. The Spearman's correlation coefficient was used to assess associations between measured parameters determined by the two methods. According to Bland and Altman [16], the agreement between two measurements were assessed by determining the mean $\pm 2 \mathrm{SD}$ of the between measurement differences. A two-sided $P$ value $<0.05$ was considered significant.

\section{Results}

Study patients

Table 1 shows the patients' characteristics. The mean age was $60.1 \pm 8.6$ years, and $57.6 \%$ of participants
Table 1 Study population

\begin{tabular}{ll}
\hline & $N=57$ \\
\hline Age, years & $60.1 \pm 8.6$ \\
Male & $34(57.6)$ \\
Diabetes & $16(27.1)$ \\
Hypertension & $30(50.8)$ \\
Current smoker & $13(22.0)$ \\
Hypercholesterolemia & $34(57.6)$ \\
Total cholesterol, mg/dl & $190.9 \pm 40.2$ \\
LDL cholesterol, mg/dl & $111.5 \pm 38.5$ \\
hsCRP (mg/l) & $2.2 \pm 3.3$ \\
Study vessel & \\
LAD & $33(55.9)$ \\
LCX & $6(10.2)$ \\
RCA & $20(33.9)$ \\
Plaque burden, \% & $60.7 \pm 0.8$ \\
\hline
\end{tabular}

Values are presented as number $(\%)$ or mean $\pm \mathrm{SD}$. Hypercholesterolemia defined as $\geq 180 \mathrm{mg} / \mathrm{dl}$ of total cholesterol

$L A D$ left anterior descending artery, $L C X$ left circumflex artery, $R C A$ right coronary artery, $h s C R P$ high-sensitivity C-reactive protein

were men. The study vessels were the left anterior descending artery in $55.9 \%$, the left circumflex artery in $10.2 \%$ and the right coronary artery in $33.9 \%$ of cases. The length of the segments was $10.2 \pm 0.3 \mathrm{~mm}$ with a volumetric plaque burden of $60.7 \pm 0.8 \%$ (43.1-75.5\%). There was no complication related to IVUS imaging.

Comparison between the conventional and Shin's methods

By design, the mean length of analysis and the mean vessel area are the same (Table 2). There were no significant differences in the mean area and volume of necrotic core and dense calcium between the two methods. The correlation coefficients $(R)$ were 0.99 $(P<0.001)$ for all compared parameters (Fig. 3). Between methods, the absolute differences in mean area and volume of necrotic core were $0.02 \pm 0.02$ $\mathrm{mm}^{2}$ and $0.34 \pm 0.29 \mathrm{~mm}^{3}$, respectively, while for mean area and volume of dense calcium, the absolute differences were $0.04 \pm 0.07 \mathrm{~mm}^{2}$ and $0.36 \pm 0.52$ $\mathrm{mm}^{3}$, respectively. Although it did not achieve statistical significance, mean dense calcium was consistently smaller and mean necrotic area was 
Table 2 Mean area and volume of necrotic core and dense calcium with conventional and Shin's method

\begin{tabular}{lccc}
\hline & Conventional & Shin's & Absolute $\triangle$ \\
\hline Frame $(n=1401)$ & & & $P$ \\
Vessel area, mm ${ }^{2}$ & $13.78 \pm 3.90$ & $13.78 \pm 3.90$ & 0 \\
DC area, $\mathrm{mm}^{2}$ & $0.41 \pm 0.51$ & $0.38 \pm 0.48$ & $0.04 \pm 0.07$ \\
NC area, $\mathrm{mm}^{2}$ & $0.78 \pm 0.70$ & $0.80 \pm 0.71$ & $0.02 \pm 0.02$ \\
Segment $(n=59)$ & & & 0.07 \\
Lesion length, mm & $10.15 \pm 0.29$ & $10.15 \pm 0.29$ & 0 \\
Vessel volume, mm $\mathrm{mm}^{3}$ & $140.65 \pm 37.90$ & $140.65 \pm 37.90$ & $0.36 \pm 0.52$ \\
DC volume, $\mathrm{mm}^{3}$ & $4.35 \pm 4.43$ & $3.99 \pm 4.16$ & $0.34 \pm 0.29$ \\
NC volume, $\mathrm{mm}^{3}$ & $8.26 \pm 6.62$ & $8.39 \pm 6.72$ & 0.66 \\
\hline
\end{tabular}

Values are presented as mean $\pm \mathrm{SD}$

$D C$ dense calcium, $N C$ necrotic core, $P$ conventional versus Shin's method, $\triangle$ difference
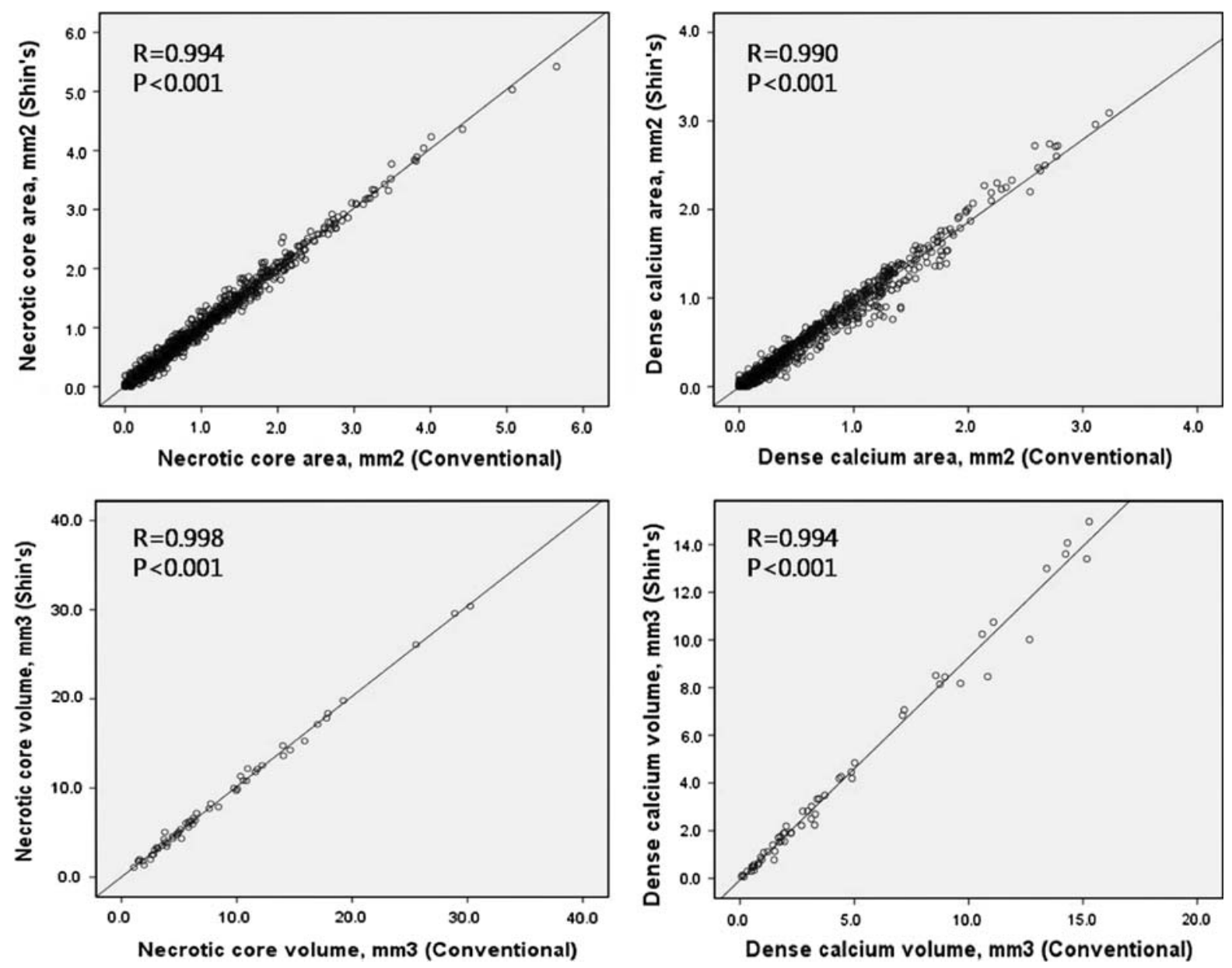

Fig. 3 Graphs show significant correlations for the absolute measurement values of each VH-IVUS plaque component between the conventional method and Shin's method for atherosclerotic coronary lesions 


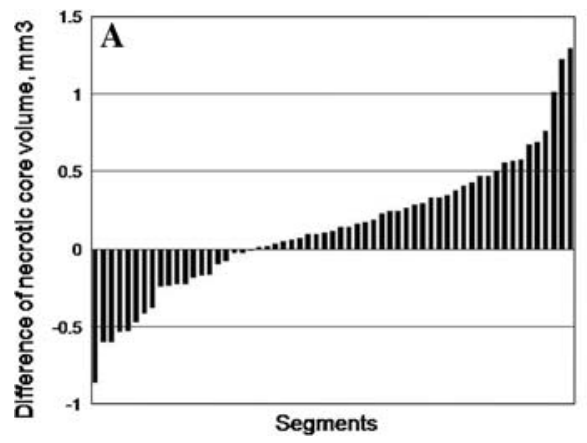

Fig. 4 Differences of necrotic core and dense calcium volumes (Shin's method minus conventional method). Mean necrotic core volumes were larger in Shin's method as

larger in Shin's method as compared to the conventional method (Fig. 4). A total of three segments in necrotic core and five segments in dense calcium had an absolute difference in volume of more than $1 \mathrm{~mm}^{3}$. The correlation of the mean areas and volumes of necrotic core and dense calcium between Shin's and the conventional method was expressed by a linear least-squares fit in Fig. 3.

Although fibrous and fibrofatty components are not correct by Shin's method because this method can not differentiate between lumen contents (usually characterized as fibrous and fibrofatty) and the fibrous and fibrofatty that are part of the plaque contents, here we provide a comparison between conventional vs. Shin's method: $3.37 \pm 2.03 \mathrm{~mm}^{2}$ vs. $4.04 \pm 2.16$ $\mathrm{mm}^{2}, P<0.001$ for fibrous area and $0.87 \pm 0.93$ $\mathrm{mm}^{2}$ vs. $1.68 \pm 1.49 \mathrm{~mm}^{2}, P<0.001$ for fibrofatty area, respectively.

\section{Reproducibility of Shin's method}

Table 3 shows the reproducibility of Shin's method. The linear least-squares fit of the relationship between the two measurements for necrotic core area and dense calcium area were $R=0.999, P<0.001$ and $R=1.0$, $P<0.001$, respectively (Fig. 5). For areas of the necrotic core and dense calcium, the means of the differences between the two measurements were $0.004 \pm 0.019$ and $0.002 \pm 0.013 \mathrm{~mm}^{2}$. The limits of agreement for area of the necrotic core and dense calcium were -0.034 to $0.042 \mathrm{~mm}^{2}$ and -0.024 to $0.051 \mathrm{~mm}^{2}$. The reproducibility coefficients were within $1 \%$ of the means of the two measurements for area and volume of the necrotic core and dense calcium.

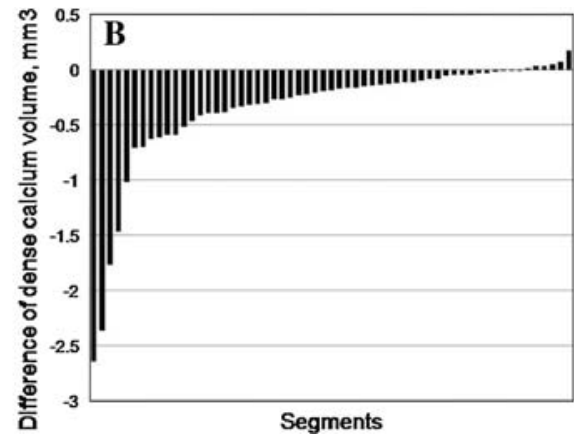

compared to the conventional method (A), while the mean dense calcium volume was consistently smaller in Shin's method as compared to the conventional method (B)

Analysis time

To assess the measurement analysis time difference between the two methods, 12 segments were randomly selected. Manual correction of the automatic contour detection was required in almost all frames for a total analysis time of $26.8 \pm 6.7 \mathrm{~min} / \mathrm{segment}$ in the conventional method. However, analysis time was $3.3 \pm 0.6 \mathrm{~min} /$ segment for Shin's method (Table 4).

\section{Discussions}

This report introduces a new measurement method (Shin's method) for necrotic core and dense calcium using VH-IVUS. The main findings of this study are: (1) necrotic core and dense calcium areas and volumes determined by Shin's method show a good correlation to those derived from the conventional method; (2) Shin's method has a high reproducibility, and (3) analysis time is shorter for Shin's method.

The introduction of RF-based IVUS analysis permits quantitative assessment of atherosclerotic plaque composition [17]. This is of crucial relevance because necrotic core is a key determinant of plaque vulnerability. This imaging modality has been extensively scrutinized and studied in different clinical contexts, and most studies using this technique have shown consistency as compared with previous pathological studies [13, 14]. However, this imaging technique is very much influenced by the precision of contour detection, which in turn is highly timeconsuming thus rendering its clinical use for online clinical decisions today not possible and not widely 
Table 3 Reproducibility of necrotic core and dense calcium by Shin's method

\begin{tabular}{|c|c|c|c|c|}
\hline & Shin's 1 & Shin's 2 & Absolute $\triangle$ & $P$ \\
\hline \multicolumn{5}{|l|}{ Frame $(n=189)$} \\
\hline Vessel area, $\mathrm{mm}^{2}$ & $14.66 \pm 3.73$ & $14.66 \pm 3.73$ & 0 & \\
\hline $\mathrm{DC}$ area, $\mathrm{mm}^{2}$ & $0.39 \pm 0.48$ & $0.38 \pm 0.48$ & $0.01 \pm 0.01$ & 0.96 \\
\hline $\mathrm{NC}$ area, $\mathrm{mm}^{2}$ & $0.82 \pm 0.57$ & $0.81 \pm 0.56$ & $0.01 \pm 0.02$ & 0.95 \\
\hline \multicolumn{5}{|l|}{ Segment $(n=8)$} \\
\hline Lesion length, mm & $10.24 \pm 0.28$ & $10.24 \pm 0.28$ & 0 & \\
\hline Vessel volume, $\mathrm{mm}^{3}$ & $150.42 \pm 35.2$ & $150.42 \pm 35.2$ & 0 & \\
\hline DC volume, $\mathrm{mm}^{3}$ & $4.03 \pm 3.78$ & $4.00 \pm 3.78$ & $0.03 \pm 0.02$ & 0.99 \\
\hline $\mathrm{NC}$ volume, $\mathrm{mm}^{3}$ & $8.39 \pm 4.58$ & $8.35 \pm 4.57$ & $0.06 \pm 0.06$ & 0.99 \\
\hline
\end{tabular}

Values are presented as mean $\pm \mathrm{SD}$

$D C$ dense calcium, $N C$ necrotic core, $P$ conventional versus Shin's method, $\triangle$ difference
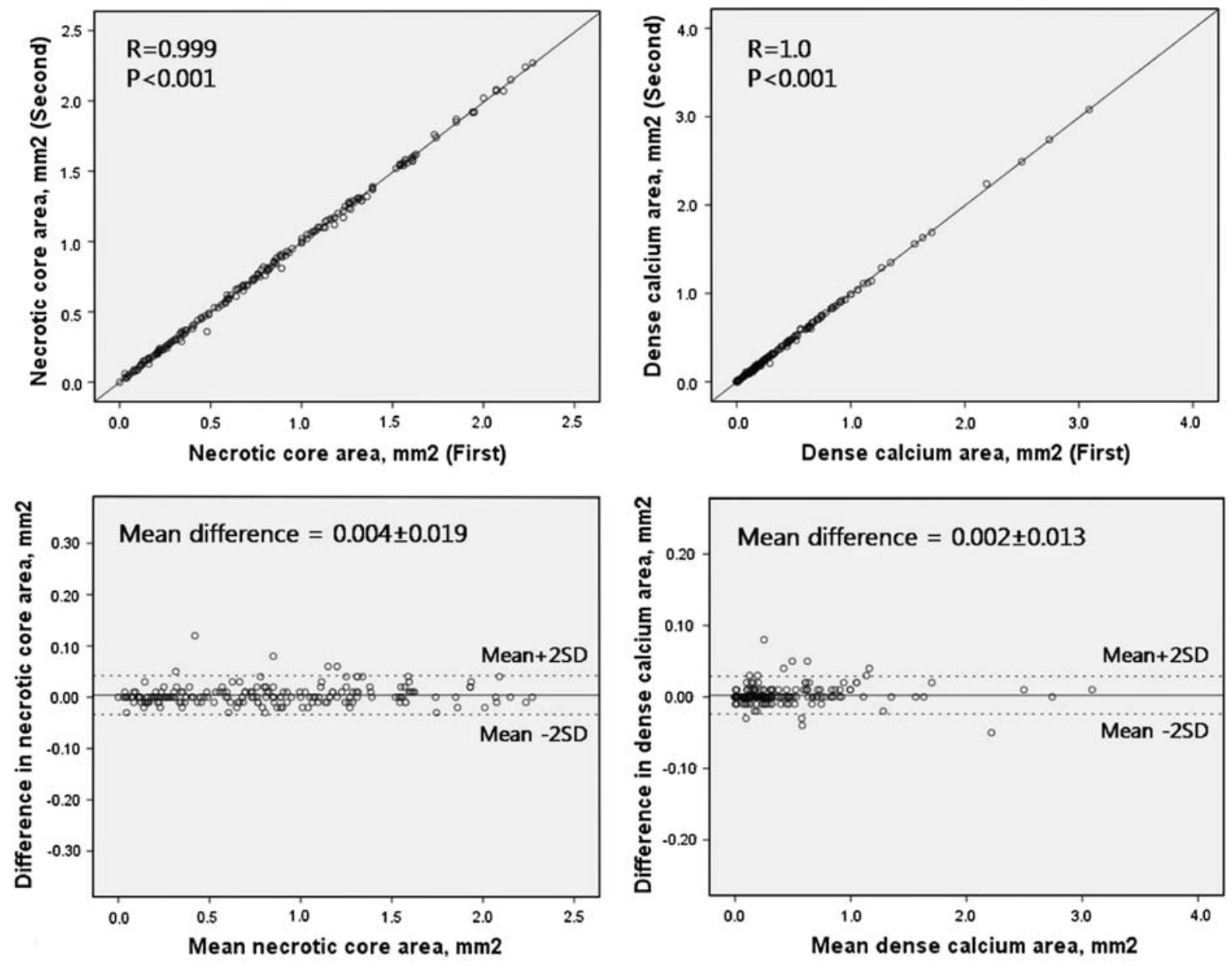

Fig. 5 Assessment of Shin's method reproducibility. The differences of the mean necrotic core and the mean dense calcium areas between the two measurements were nearly zero,

and the reproducibility coefficients were within $1 \%$ of the means of the two measurements 
Table 4 Comparison of analysis time between the conventional method and Shin's method (12 segments)

\begin{tabular}{llccc}
\hline & Conventional & Shin's & Absolute $\triangle$ & $P$ \\
\hline Analyzed length, mm & $10.1 \pm 0.2$ & $10.1 \pm 0.2$ & 0 & 1.0 \\
Analysis time, min & $26.8 \pm 6.7$ & $3.3 \pm 0.6$ & $23.5 \pm 6.2$ & $<0.001$ \\
\hline
\end{tabular}

Values are presented as mean \pm SD. $P$ Conventional vs. Shin's method, $\triangle$ difference

accepted. There was a need therefore to explore a different manner of necrotic core quantification that would allow information to be quickly obtained as accurate as possible and with excellent reproducibility.

Use of Shin's method does not require training, and more rapid contour detection around the catheter can be done. In the future, with the implementation of an automatic catheter contour detection program, no user intervention will be needed. This catheter contour must be drawn beyond the ring-down artifact (i.e., 'flare') so that this is not misclassified as necrotic core or dense calcium.

Although in the conventional method we would have included thrombi that are undetected by IVUS, recent papers showed that thrombus was always classified as fibrous and fibrofatty tissues by VH-IVUS $[18,19]$, and therefore even when the luminal contour would have included some thrombi, the necrotic core and calcium measurement using Shin's method is not affected by a variable contour detection.

Although this study showed that the sum of necrotic core and calcium was very similar using both methods, the mean dense calcium area was consistently smaller and the mean necrotic area was larger in Shin's method as compared to the conventional method. In particular, a total of five segments in dense calcium and three segments in necrotic core had an absolute difference in volume of more than $1 \mathrm{~mm}^{3}$. Possible explanations for this difference between two methods are as follows: (1) Thin plaque is treated as a grey medial stripe by VH-IVUS in the conventional method. However, Shin's method is not influenced by the artificial thickness of the medial stripe. Therefore, Shin's method shows more necrotic core even though there is a thin plaque (Fig. 6). (2) In the case of mixed plaques at the lumen, in the conventional method, the proximity of the contour with the plaque, will characterize the adjacent tissue to the contour using one of the two classification trees of the virtual histology system, resulting in more calcium or more necrotic core at that area (Fig. 6). In
Shin's method, a longer distance will be present from the catheter contour to the plaque, and therefore the second classification tree would be applied resulting in a slightly different tissue characterization. In a previous study, in vivo VH-IVUS images suggested an increase in dense calcium in the acute coronary syndrome group when compared with the stable angina group. Although in general the in vivo VH-IVUS data correlated well with in vitro histology (predictive accuracy of $96.5 \%$ ), the RF data analysis overestimated the frequency of calcifications. The authors mentioned that one possible explanation was that the artifact is colored with white because the software for $\mathrm{VH}$ analysis is obliged to assign one of the four colors for each pixel [13].

Previous data with the conventional method showed acceptable reproducibility for plaque composition; likewise, repeated measurements for necrotic core and calcium with Shin's method were highly correlated. Awareness of inter-observer differences may be particularly important in serial pharmacological intervention trials where small changes in plaque composition (i.e., necrotic core) may be expected $[20,21]$. Therefore, Shin's method may be a more adequate system to assess the change of necrotic core in longitudinal studies.

Shin's method for measurement of necrotic core and dense calcium using VH-IVUS demonstrated a good correlation with the conventional method and excellent reproducibility. In the future, since Shin's method could be automatically applied using an automated catheter contour detection with a computer program, and analysis time will be further decreased. This would also mean that the technique can be used in the catheterization laboratory to help operators in decision making based on content of necrotic core or calcium.

\section{Limitations}

This study has several limitations. (1) Because patients in the present study all suffered unstable 

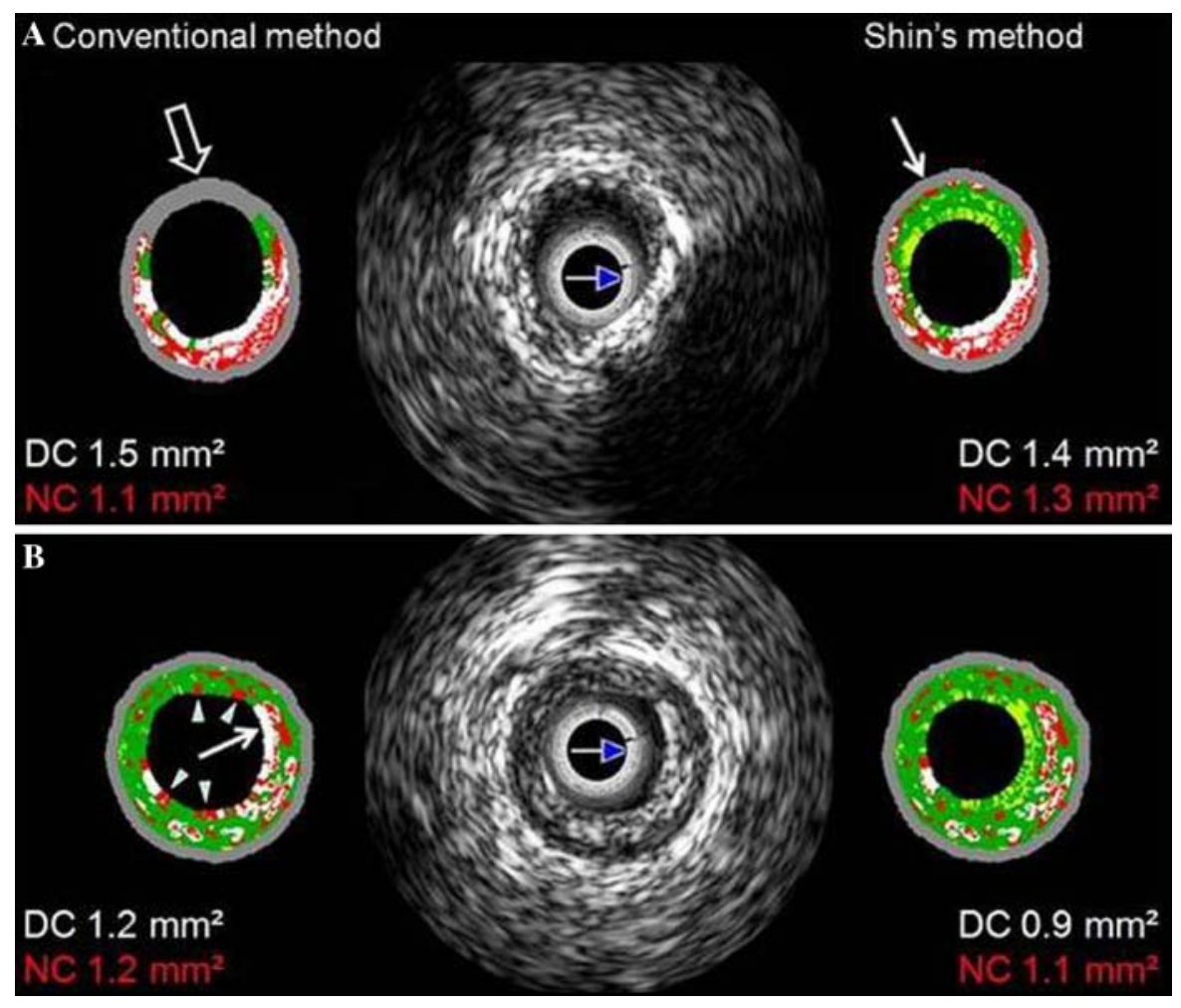

Fig. 6 A Shin's method shows some necrotic core in the grey medial stripe (white arrow) that was not shown with the conventional method (white empty arrow). B In case of superficial calcium, there is much more calcium apparent behind the luminal contour in the conventional method (white

angina, we cannot know how well the two methods would correlate in other atherosclerotic lesions. (2) The study did not include a validation component as therefore the accuracy of Shin's method remains to be determined (3) Shin's method does not show the lumen area and plaque area. Therefore, greyscale should remain the gold standard to assess plaque size changes (geometrical analysis), while virtual histology should be used to obtain compositional analysis, so as complementary information to greyscale. (4) Shin's method does not provide correct quantification of fibrous or fibrofatty tissues.

\section{Conclusions}

Shin's method for measurement of necrotic core and dense calcium using VH-IVUS demonstrated a good correlation with the conventional method and a high reproducibility. Also, Shin's method required a arrow) while in Shin's method, superficial calcium shows very finely and some decrease of calcium area. Also, some necrotic core shows behind the luminal contour (white arrow heads) in the conventional method ( $D C$ dense calcium, $N C$ necrotic core)

significantly shorter analysis time than the conventional method. Therefore, Shin's method could replace the conventional method for necrotic core and calcium measurement in atherosclerotic lesions, and it might be useful in the catheterization laboratory for online clinical decision.

Open Access This article is distributed under the terms of the Creative Commons Attribution Noncommercial License which permits any noncommercial use, distribution, and reproduction in any medium, provided the original author(s) and source are credited.

\section{References}

1. Virmani R, Burke AP, Farb A et al (2006) Pathology of the vulnerable plaque. J Am Coll Cardiol 47:C13-C18

2. Schoenhagen P, Nissen SE (2005) Assessing coronary plaque burden and plaque vulnerability: atherosclerosis imaging with IVUS and emerging noninvasive modalities. Am Heart Hosp J 1:164-169 
3. Schoenhagen P, Nissen SE (2003) Coronary atherosclerotic disease burden: an emerging endpoint in progression/ regression studies using intravascular ultrasound. Curr Drug Targets Cardiovasc Haematol Disord 3:218-226

4. Schaar JA, de Korte CL, Mastik F et al (2003) Intravascular palpography for high-risk vulnerable plaque assessment. Herz 28:488-495

5. Van Mieghem CA, McFadden EP, de Feyter PJ et al (2006) Noninvasive detection of subclinical coronary atherosclerosis coupled with assessment of changes in plaque characteristics using novel invasive imaging modalities: the Integrated Biomarker and Imaging Study (IBIS). J Am Coll Cardiol 47:1134-1142

6. Garcia-Garcia HM, Gonzalo N, Regar E et al (2009) Virtual histology and optical coherence tomography: from research to a broad clinical application. Heart 95: 1362-1374

7. Valgimigli M, Rodriguez-Granillo GA, Garcia-Garcia HM et al (2006) Distance from the ostium as an independent determinant of coronary plaque composition in vivo: an intravascular ultrasound study based radiofrequency data analysis in humans. Eur Heart J 27:655-663

8. Rodriguez-Granillo GA, Garcia-Garcia HM, Valgimigli M et al (2006) Global characterization of coronary plaque rupture phenotype using three-vessel intravascular ultrasound radiofrequency data analysis. Eur Heart J 27: 1921-1927

9. Mehta SK, McCrary JR, Frutkin AD et al (2007) Intravascular ultrasound radiofrequency analysis of coronary atherosclerosis: an emerging technology for the assessment of vulnerable plaque. Eur Heart J 28:1283-1288

10. Konig A, Klauss V (2007) Virtual histology. Heart 93: 977-982

11. Hong M-K, Mintz GS, Lee CW et al (2007) Comparison of virtual histology to intravascular ultrasound of culprit coronary lesions in acute coronary syndrome and target coronary lesions in stable angina pectoris. The American Journal of Cardiology 100:953-959

12. Fujii K, Carlier SHG, Mintz GS et al (2005) Association of plaque characterization by intravascular ultrasound virtual histology and arterial remodeling. The American Journal of Cardiology 96:1476-1483

13. Nasu K, Tsuchikane E, Katoh O et al (2006) Accuracy of in vivo coronary plaque morphology assessment: a validation study of in vivo virtual histology compared with in vitro histopathology. J Am Coll Cardiol 47:2405-2412

14. Nair A, Kuban BD, Tuzcu EM et al (2002) Coronary plaque classification with intravascular ultrasound radiofrequency data analysis. Circulation 106:2200-2206

15. Braunwald E, Antman EM, Beasley JW et al (2002) ACC/ AHA guideline update for the management of patients with unstable angina and non-ST-segment elevation myocardial infarction-2002: summary article: a report of the American College of Cardiology/American Heart Association Task Force on Practice Guidelines (committee on the management of patients with unstable Angina). Circulation 106:1893-1900

16. Bland JM, Altman DG (1986) Statistical methods for assessing agreement between two methods of clinical measurement. Lancet 1:307-310

17. Mintz GS, Maehara A (2009) Serial intravascular ultrasound assessment of atherosclerosis progression and regression. Circ J 73:1557-1560

18. Nasu K, Tsuchikane E, Katoh O et al (2008) Impact of intramural thrombus in coronary arteries on the accuracy of tissue characterization by in vivo intravascular ultrasound radiofrequency data analysis. Am J Cardiol 101: 1079-1083

19. Frutkin AD, Mehta SK, McCrary JR et al (2007) Limitations to the use of virtual histology-intravascular ultrasound to detect vulnerable plaque. Eur Heart J 28:1783-1784

20. Serruys PW, Garcia-Garcia HM, Buszman P et al (2008) Effects of the direct lipoprotein-associated phospholipase A2 inhibitor darapladib on human coronary atherosclerotic plaque. Circulation 118:1172-1182

21. Kawasaki M, Sano K, Okubo M et al (2005) Volumetric quantitative analysis of tissue characteristics of coronary plaques after statin therapy using three-dimensional integrated backscatter intravascular ultrasound. J Am Coll Cardiol 45:1946-1953 